\title{
Thermodynamic Properties of $(\mathrm{Ca}, \mathrm{Sr})_{2} \mathrm{RuO}_{4}$ in Magnetic Fields
}

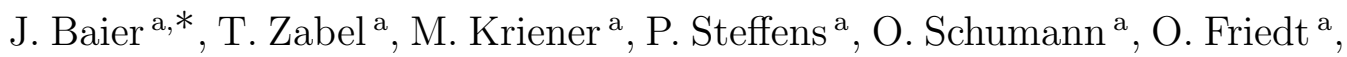

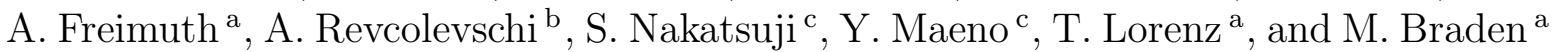 \\ ${ }^{\mathrm{a}}$ II. Physikalisches Institut, University of Cologne, 50937 Cologne, Germany \\ ${ }^{\mathrm{b}}$ Lab. de Physico-Chimie de l'État Solide, Université Paris-Sud, France \\ ${ }^{\mathrm{c}}$ Department of Physics, Kyoto University, Kyoto 606-8502, Japan
}

\begin{abstract}
We have studied the influence of a magnetic field on the thermodynamic properties of $\mathrm{Ca}_{2}-x \mathrm{Sr}_{x} \mathrm{RuO}_{4}$ in the intermediate metallic region with tilt and rotational distortions $(0.2 \leq x \leq 0.5)$. We find strong and anisotropic thermal expansion anomalies at low temperatures, which are suppressed and even reversed by a magnetic field. The metamagnetic transition of $\mathrm{Ca}_{1 .} \mathrm{Sr}_{0.2} \mathrm{RuO}_{4}$ is accompanied by a large magnetostriction. Furthermore, we observe a strong magnetic-field dependence of $c_{p} / T$, that can be explained by magnetic fluctuations.
\end{abstract}

Key words: ruthenates, layered perovskite, specific heat, thermal expansion, heavy fermions, metamagnetism, electronic correlations PACS: 65.40.-b, 65.40.Ba, 65.40.De, 71.27.+a, 75.30.Kz

The phase diagram of $\mathrm{Ca}_{2-x} \mathrm{Sr}_{x} \mathrm{RuO}_{4}$ possesses a rich spectrum of structural distortions accompanied by changes of the physical properties $[1,2,3]$. The spintriplet superconductor $\mathrm{Sr}_{2} \mathrm{RuO}_{4}$ is driven to the antiferromagnetic Mott insulator $\mathrm{Ca}_{2} \mathrm{RuO}_{4}$. With decreasing $\mathrm{Sr}$ content, the $\mathrm{K}_{2} \mathrm{NiF}_{4}$ structure of pure $\mathrm{Sr}_{2} \mathrm{RuO}_{4}$ gets more and more distorted. First, a rotation of the $\mathrm{RuO}_{6}$ octahedra around the $c$ axis occurs. This rotational distortion comes along with an increase of the low-T susceptibility $\chi$, that is about a factor 200 larger at $x=0.5$ than that of $\mathrm{Sr}_{2} \mathrm{RuO}_{4}[1]$. Below $x=0.5$ an additional tilt of the octahedra around an in-plane axis occurs [3] and the low-field magnetization at low temperatures is reduced again. In the tilted phase, at $x=$ 0.2 , a metamagnetic transition at $H_{c} \simeq 5 \mathrm{~T}$ is observed, leading to a high-field magnetization which exceeds that of $\mathrm{Ca}_{1.5} \mathrm{Sr}_{0.5} \mathrm{RuO}_{4}$ [4]. The variation of the low- $\mathrm{T}$ specific heat divided by temperature $c_{p} / T$ as a function of Ca content resembles that of $\chi$. First, $c_{p} / T$ increases upon Ca-doping and reaches an unusually high value at $x=0.5\left(c_{p} / T \simeq 255 \mathrm{~mJ} / \mathrm{mole} \mathrm{Ru} \cdot \mathrm{K}^{2}\right)$, that

\footnotetext{
* Corresponding author

Email address: baier@ph2.uni-koeln.de (J. Baier).
}

lies in the range of typical heavy fermion compounds [4]. Upon further increase of the Ca content, $c_{p} / T$ again decreases and shows a non-monotonous temperature dependence at low temperatures for $x=0.2$.

Here, we present measurements of the magnetostriction and the magnetic-field dependence of thermal expansion and specific heat of two single crystals, $\mathrm{Ca}_{1.8} \mathrm{Sr}_{0.2} \mathrm{RuO}_{4}(x=0.2)$ and $\mathrm{Ca}_{1.5} \mathrm{Sr}_{0.5} \mathrm{RuO}_{4}(x=$ $0.5)$, that were grown by a floating zone technique at Kyoto University $(x=0.2)$ and at the Université of Paris Sud $(x=0.5)$. Magnetostriction and thermal expansion were recorded by high-resolution capacitive dilatometry and the specific heat was measured between $300 \mathrm{mK}$ and $60 \mathrm{~K}$ by a home-built Nernst calorimeter based on a commercial ${ }^{3} \mathrm{He}$ refrigerator. The measurements were carried out in magnetic fields up to $14 \mathrm{~T}$. $\mathrm{Ca}_{1.8} \mathrm{Sr}_{0.2} \mathrm{RuO}_{4}$ exhibits a large magnetostriction with a steep increase around $6 \mathrm{~T}$ at the metamagnetic transition at lowest temperatures (see fig.1, left panel). The length change has been recorded parallel to the $c$ axis, in a longitudinal applied field. On the same sample we find an anomalous thermal expansion (see fig.1, right panel). Upon cooling below $25 \mathrm{~K}$, a strong compression of the $c$ axis sets in. This 


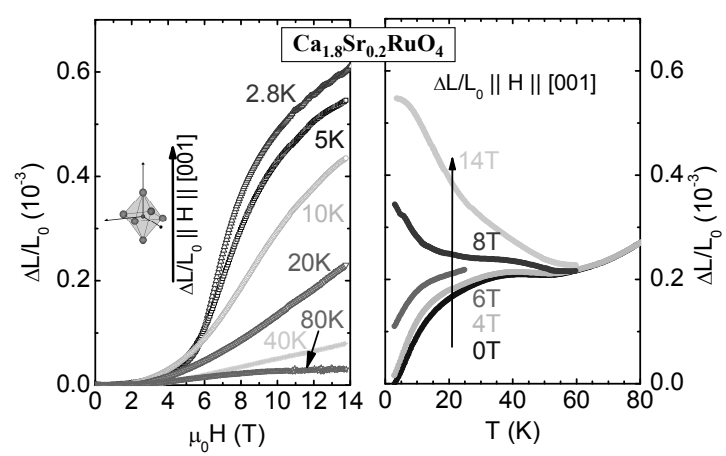

Fig. 1. Magnetostriction (left) and thermal expansion (right) of $\mathrm{Ca}_{1.8} \mathrm{Sr}_{0.2} \mathrm{RuO}_{4}(x=0.2)$. The length change was recorded parallel to the $c$ axis in a longitudinal magnetic field.

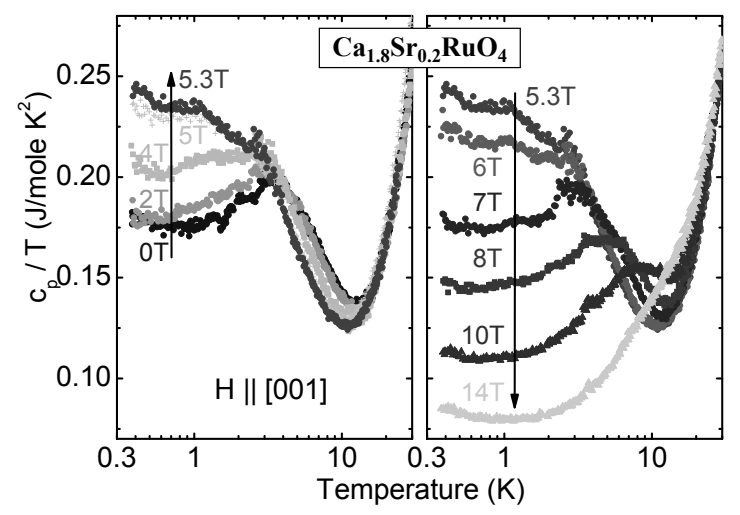

Fig. 2. Specific heat divided by temperature $c_{p} / T$ of $\mathrm{Ca}_{1.8} \mathrm{Sr}_{0.2} \mathrm{RuO}_{4}$ in magnetic fields below (left) and above (right) the critical field of the metamagnetic transition.

anomalous compression is systematically reduced by magnetic fields with $H \leq 6 \mathrm{~T}$. Above $6 \mathrm{~T}$, this compression turns into a $c$ axis expansion. Measurements of the thermal expansion perpendicular to the $c$ axis (not shown) reveal an opposed behavior, with the $a b$ plane shrinking upon cooling below $25 \mathrm{~K}$ [5]. A similar thermal expansion anomaly is also observed beyond the tilted phase for samples with $x=0.5,0.62$ and 1 , but gets more pronounced with decreasing Sr content [5]. This low-T thermal expansion anomaly and the effect of the magnetic field have to be attributed to an electronic origin. A detailed discussion and the results of neutron diffraction experiments on the same samples have been presented in a previous study [5].

We observe also a strong field-dependence of the low$\mathrm{T}$ specific heat. As mentioned above, without magnetic field, $c_{p} / T$ is reduced for $x=0.2$ in comparison with $x=0.5$. Additionally, it possesses a non-monotonous temperature dependence, with a maximum at about $5 \mathrm{~K}$. As presented in fig. 2, the application of a magnetic field leads first to an increase of the low- $T$ value of $c_{p} / T$. At a field of $5.3 \mathrm{~T}$, in the vicinity of the metamagnetic transition, it reaches a maximum value close

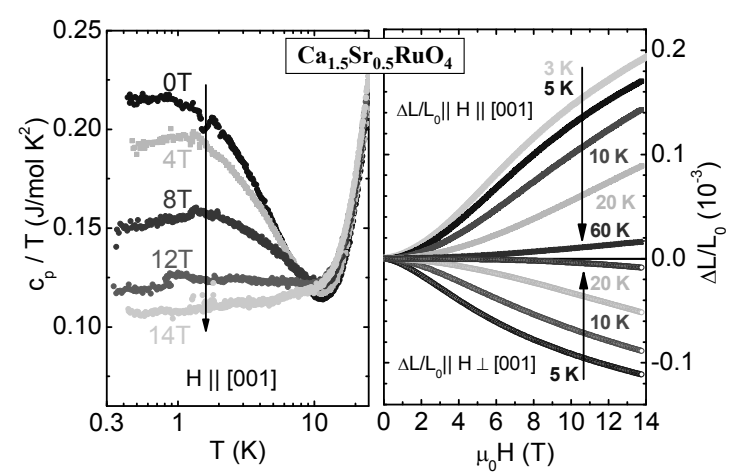

Fig. 3. Left: $c_{p} / T$ of $\mathrm{Ca}_{1.5} \mathrm{Sr}_{0.5} \mathrm{RuO}_{4}$ in magnetic fields applied parallel to the $c$ axis. Right: Magnetostriction of $\mathrm{Ca}_{1.5} \mathrm{Sr}_{0.5} \mathrm{RuO}_{4}$ parallel and perpendicular to the $c$ axis in a longitudinal applied field in each case.

to the $c_{p} / T$ value for $x=0.5$ at $H=0 \mathrm{~T}$. A further increase of the magnetic field leads again to a decrease of $c_{p} / T$. This suggests that the enhancement of $c_{p} / T$ at the metamagnetic transition is due to magnetic fluctuations that are suppressed by the magnetic field in the spin-polarized phase at $H>H_{c}$. Recently, it was shown by inelastic neutron scattering experiments on $\mathrm{Ca}_{1.38} \mathrm{Sr}_{0.62} \mathrm{RuO}_{4}$, that there are strong magnetic fluctuations in this compound [6].

$\mathrm{Ca}_{1.5} \mathrm{Sr}_{0.5} \mathrm{RuO}_{4}$ shows a qualitatively similar magnetostriction, which, however, is smaller and lacks the steep increase. The $c$ axis expands and the $a b$ plane shrinks in a longitudinal applied magnetic field (see fig. 3, right panel). The specific heat measurements provide a monotonic decrease of $c_{p} / T$ at low temperatures as a function of magnetic field (see fig. 3, left panel), similar that of $\mathrm{Ca}_{1.8} \mathrm{Sr}_{0.2} \mathrm{RuO}_{4}$ at $H>H_{c}$. These findings agree with the idea that the increase of the $\mathrm{Sr}$ concentration suppresses the metamagnetic transition field; from $\simeq 5 \mathrm{~T}$ for $x=0.2$ it goes down close to zero for $x=0.5$.

In summary, we have found a large magnetostriction and a strong effect of the magnetic field on the low temperature specific heat. These data point to strong magnetic fluctuations around the metamagnetic transition in $\mathrm{Ca}_{2-x} \mathrm{Sr}_{x} \mathrm{RuO}_{4}$.

This work was supported by the DFG via SFB 608 .

\section{References}

[1] S. Nakatsuji and Y. Maeno, Phys. Rev. Lett. 84, 2666 (2000).

[2] S. Nakatsuji and Y. Maeno, Phys. Rev. B 62, 6458 (2000).

[3] O. Friedt et al., Phys. Rev. B 63, 174432 (2001).

[4] S. Nakatsuji et al., Phys. Rev. Lett. 90, 137202 (2003).

[5] M. Kriener et al., cond-mat 0408015, (2004). 
[6] O. Friedt et al., Phys. Rev. Lett. 93, 147404 (2004). 